Check for updates

Cite this: RSC Adv., 2017, 7, 34508

\title{
Porous Li-containing biphasic calcium phosphate scaffolds fabricated by three-dimensional plotting for bone repairł
}

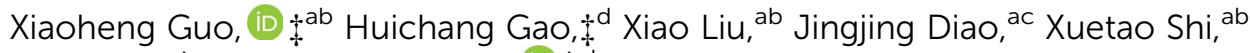 \\ Naru Zhao*ac and Yingjun Wang (D) *ab
}

A well-designed scaffold with accurately controlled structure, pore size as well as interconnection plays a key role in tissue engineering. In this study, porous Li-containing biphasic calcium phosphate scaffolds (Li-BCP) with expected structure were fabricated successfully by 3D plotting technique. The results of scanning electron microscopy (SEM) and micro-CT indicated that scaffolds from 3D plotting possessed an elaborate pore structure, porosity and interconnection. Besides, Li-BCP scaffolds also possessed excellent mechanical strength. Furthermore, in vitro cell experiments confirmed that mouse bone mesenchymal stem cells (mBMSCs) cultured on Li-BCP scaffolds exhibited good adhesion and spreading. Moreover, Li-BCP scaffolds could efficaciously promote the proliferation and osteogenic differentiation of mBMSCs, demonstrating their potential applicability as a candidate for bone repair applications.

Received 12th April 2017

Accepted 30th June 2017

DOI: $10.1039 / \mathrm{c} 7 \mathrm{ra0} 4155 \mathrm{a}$

rsc.li/rsc-advances

assemble a physical model at a scale according to a pre-

\section{Introduction}

The development of tissue engineering prompts a huge opportunity for the repair and regeneration of bone defects. ${ }^{1}$ Porosity is widely regarded as a prerequisite for osteogenesis, and porous three-dimensional (3D) biomaterials have been used extensively as scaffolds in the field of tissue engineering. ${ }^{2}$ As an integral part of bone tissue engineering, 3D porous scaffolds can mimic the extracellular matrix (ECM) properties as well as be favorable for cell attachment and stimulate bone tissue formation in vivo. ${ }^{3}$ In order to fabricate these $3 \mathrm{D}$ porous scaffolds, a variety of conventional methods including gas foaming, phase separation, melt molding, freeze drying and particulate leaching have been utilized. ${ }^{2,4}$ However, some defects of these conventional methods, such as the poor control of pore size, interconnection and geometry, are a serious constraint to their application and development. ${ }^{5}$ Fortunately, the emerging rapid prototyping (RP) techniques in recent years can perfectly overcome these deficiencies, which could quickly fabricate or

${ }^{a}$ School of Materials Science and Engineering, South China University of Technology, Guangzhou 510641, China. E-mail: nrzhao@scut.edu.cn; imwangyj@163.com; Tel: +86-20-87114645; +86-20-22236066

${ }^{b}$ Nation Engineering Research Centre for Tissue Restoration and Reconstruction, Guangzhou 510006, China

${ }^{c}$ Guangdong Province Key Laboratory of Biomedical Engineering, South China University of Technology, Guangzhou 510006, China

${ }^{d}$ School of Medicine, South China University of Technology, Guangzhou 510006, China $\dagger$ Electronic supplementary information (ESI) available. See DOI: $10.1039 / \mathrm{c} 7 \mathrm{ra} 04155 \mathrm{a}$

$\ddagger$ These authors contributed equally to this work. designed computer-assisted design (CAD) model. ${ }^{6,7}$

3D plotting is one type of versatile RP technique to prepare porous scaffolds of tissue engineering, which could not only provide the ideal pore size and interconnection, but also guarantee the requirements of personalized customization. ${ }^{8}$ In addition, all the procedures of 3D plotting are conducted under mild conditions, which makes it possible for all of the biological components such as proteins and even living cells remain active during the 3D plotting process. ${ }^{9}$

Selecting suitable raw materials for 3D plotting scaffolds is an essential part in bone repair. To date, a wide range of materials have been successfully applied to 3D plotting such as polycaprolactone (PCL), ${ }^{4,7}$ hydrogel, ${ }^{10}$ calcium phosphate cement (CPC), ${ }^{11}$ polylactic-co-glycolic acid (PLGA), ${ }^{12}$ gelatinalginate composites, ${ }^{6,13}$ PCL-hydroxyapatite (HA) composites, ${ }^{8}$ bioactive glass-alginate composites, ${ }^{9}$ HA-alginate composites,${ }^{14}$ CPC-alginate composites, ${ }^{15}$ chitosan-hydrogel composites etc. ${ }^{16}$ However, there are a few reports that using $3 \mathrm{D}$ plotting RP technique to fabricate bioceramics scaffolds. Bioactive ceramics, such as $\beta$-tricalcium phosphate ( $\beta$-TCP) and HA, have been proven to be ideal candidates for bone repair due to their homologous chemical constitution with the natural bone. ${ }^{17}$ However, the application of bioactive ceramics is highly restrained, as the rate between degradation of bioactive ceramics and formation of fresh bone tissues does not match. Fortunately, regulating HA/TCP ratios of synthesized biphasic calcium phosphate (BCP) can contribute to scaffold degradation rate, therefore the rate between materials degradation and new bone growth could eventually reach a balance, meanwhile 
effectively promote bone ingrowth and vascularization in vivo. ${ }^{18-22}$ However, bioactive ceramics are still far away from ideal bone repair materials due to their limited bone induction ability. ${ }^{23}$

In recent years, there has been an increasing amount of reports that focus on the incorporation of functional trace metallic elements into calcium phosphate (CPs) to improve its biological performance. Metallic ions (for instance, $\mathrm{Li}^{+},{ }^{23} \mathrm{Na}^{+},{ }^{24}$ $\mathrm{Mg}^{2+},{ }^{25} \mathrm{Zn}^{2+},{ }^{26} \mathrm{Si}^{4+},{ }^{27} \mathrm{Sr}^{2+},{ }^{28}$ etc.) are indispensable to the physiological metabolism in human body. When incorporated into CPs, metallic ions can effectively improve the degradation rate of CPs, increase the mechanical strength of the materials, and stimulate some positively biological responses. ${ }^{29}$ Among those metallic ions, $\mathrm{Li}^{+}$, as a relatively new additive, catched the attention of researchers due to its excellent ability of fracture healing and osteogenesis. ${ }^{30}$ Moreover, $\mathrm{Li}^{+}$has also been proven to improve thermal stability and crystal stabilization when incorporated into $\mathrm{TCP},{ }^{24}$ and has a potential application in tissue repair.

Herein, this paper aims to construct 3D porous Li-containing BCP scaffolds (Li-BCP) with desired structure and pore size by 3D plotting technique. The structure, morphology, porosity, connectivity and mechanical properties of scaffolds were studies in detail. Moreover, we also evaluated the in vitro cell behaviors including the adhesion, spreading, proliferation, osteogenic differentiation of mouse bone mesenchymal stem cells (mBMSCs) on Li-BCP scaffolds. It was expected that the Li-BCP scaffolds will have great potential for bone repair application.

\section{Experimental section}

\section{Materials}

The starting materials used in this study included calcium nitrate $\left(\mathrm{Ca}\left(\mathrm{NO}_{3}\right)_{2} \cdot 4 \mathrm{H}_{2} \mathrm{O}\right.$, Guangzhou Chemical Co., China), diammonium phosphate $\left(\left(\mathrm{NH}_{4}\right)_{2} \mathrm{HPO}_{4}\right.$, Guangzhou Chemical Co., China), polyethylene glycol (PEG-6000, Guangzhou Chemical Co., China), glycerol $\left(\mathrm{C}_{3} \mathrm{H}_{8} \mathrm{O}_{3}\right.$, Guangzhou Chemical Co., China), n-octanol $\left(\mathrm{C}_{8} \mathrm{H}_{18} \mathrm{O}\right.$, Guangzhou Chemical Co., China), lithium chloride (LiCl, Aladdin, USA), hydroxypropyl methyl cellulose (HPMC-4000, Aladdin, USA), polyacrylamide (PAA$\mathrm{NH}_{4}$, Adamas, China).

\section{Construction of 3D plotting scaffolds}

Prior to preparation of 3D plotting scaffolds, HA, $\beta$-TCP and Licontaining $\beta$-TCP ( $\mathrm{Li}-\beta$-TCP, $\left.\left(\mathrm{Li}_{0.20} \mathrm{Ca}_{0.90}\right)_{3}\left(\mathrm{PO}_{4}\right)_{2}\right)$ were fabricated by chemical precipitation method. Briefly, $\mathrm{Ca}\left(\mathrm{NO}_{3}\right)_{2}-$ - $4 \mathrm{H}_{2} \mathrm{O}$ and $\left(\mathrm{NH}_{4}\right)_{2} \mathrm{HPO}_{4}$ were used to synthesize pure $\mathrm{HA}$ and $\beta$ TCP with the $\mathrm{Ca} / \mathrm{P}$ molar ratios fixed at 1.67 and 1.5 while the $\mathrm{pH}$ of the solution was maintained 9.5-9.8 and 6.5-6.8, respectively, during precipitation with the aid of ammonia. The synthetic process of Li- $\beta$-TCP powder was similar to $\beta$-TCP while 0.1 M Ca were replaced by $\mathrm{Li}$ (supplied by $\mathrm{LiCl}$ ).

The paste with well fluidity and suitable viscosity is a necessary condition for plotting, which directly determines the printability of scaffolds and the controllability of geometry. In this work, the pastes of Li-containing BCP (Li-BCP) and BCP scaffolds were prepared firstly. In brief, HA and Li- $\beta$-TCP with a weight ratio of $6: 4$ were as the solid content of Li-BCP scaffolds while Li- $\beta$-TCP was replaced by $\beta$-TCP in BCP scaffolds. PAA- $\mathrm{NH}_{4}(1.5 \%$ of the solid content) as a dispersant was added to deionized water/glycerol (w/w, $7 / 3$ ), and adjusted the $\mathrm{pH}=9$ of solution by $\mathrm{NH}_{3} \cdot \mathrm{H}_{2} \mathrm{O}(\mathrm{v} / \mathrm{v}, 1 / 1)$. Subsequently, the dispersant was added to a ball mill pot with solid content powder and grinded for $12 \mathrm{~h}$, then HPMC and $n$-octanol were added to the pot to get the pastes possessed appropriate viscosity and free of bubbles. In the end, the pastes were transferred to a cartridge and sonicated for $30 \mathrm{~min}$ before plotting.

The prepared pastes were used to fabricate $3 \mathrm{D}$ plotting LiBCP and BCP scaffolds. First, the cartridge with a fine nozzle $(200 \mu \mathrm{m})$ was installed to the plotter device (3D Bio-printer ${ }^{\mathrm{TM}}$ $\mathrm{V} 1.2$, China), and then a $10 \mathrm{~mm} \times 10 \mathrm{~mm} \times 5 \mathrm{~mm}$ cylindrical block model was designed by a CAD software and loaded into plotting system. The plotting speed was set as $8-13 \mathrm{~mm} \mathrm{~s}^{-1}$, the spacing between two deposited fibers was $200 \mu \mathrm{m}$, the thickness of neighboring layers was $160 \mu \mathrm{m}$ and the platform temperature was set as $42-48{ }^{\circ} \mathrm{C}$, respectively. When all the plotting parameter was set up, a nitrogen gas pressure of $0.2-0.5 \mathrm{MPa}$ was applied to the cartridge through a pressurized cap, and the cylindrical blocks with the height of $5 \mathrm{~mm}$ and a crossing angel of $90^{\circ}$ were plotted in a layer by layer manner. The obtained scaffolds were subsequently sintered at $1150{ }^{\circ} \mathrm{C}$ for $2 \mathrm{~h}$ at a heating rate of $2{ }^{\circ} \mathrm{C} \min ^{-1}$ to make the specimens densification.

\section{Characterization of scaffolds}

The phase compositions of HA, $\beta$-TCP, Li- $\beta$-TCP and scaffolds were demonstrated via X-ray diffraction (XRD, Empyrean, Netherlands) with $\mathrm{Cu} \mathrm{K} \alpha$ radiation $(\lambda=1.5418 \AA$ ) within a $2 \theta$ range of $10-60^{\circ}$ at a scan rate of $0.05^{\circ} \mathrm{min}^{-1}$ and an interval of $0.02^{\circ}$. The actual Li content of Li- $\beta$-TCP was measured by inductively coupled plasma (ICP, Optima 8300, PerkinElmer, USA). The morphology and structure of scaffolds were examined using a field-emission scanning electron microscope (FESEM, Merlin, Zeiss, Germany), prior to microscopy, the samples were coated with gold (120 s). The pore interconnectivity and porosity were characterized using micro-CT (Zkks-Mct-Sharp, Kaisheng, China) and measured by Mimics software (Materialise, Leuven, Belgium). The mechanical properties of scaffolds were determined by universal material testing machine (5967, Instron, USA), briefly, the samples were tested under axial compression at a crosshead speed of $0.5 \mathrm{~mm} \mathrm{~min}^{-1}$ until samples fragmentation, the pore channels of samples were parallel to crosshead in the test process, five parallel samples were used for each group.

\section{Cell culture}

Mouse bone mesenchymal stem cells (mBMSCs, CRL-12424, ATCC, USA) were cultured in Dulbecco's modified Eagle's medium (DMEM, Gibco, USA) with high glucose and supplemented with $10 \%$ fetal bovine serum (FBS, Life Technologies, Gibco, USA). The samples were treated with autoclave 
sterilization and placed in 48-well plates. After pretreatment with the culture medium for $6 \mathrm{~h}$, the cells were seeded at a preset density and incubated at $37{ }^{\circ} \mathrm{C}$ in a humidified incubator with $5 \% \mathrm{CO}_{2}$.

\section{Cell adhesion and spreading}

Cell adhesion was determined by observing the cell morphologies using FESEM with an accelerated voltage of $10 \mathrm{kV}$. Briefly, the mBMSCs cells $\left(2 \times 10^{4}\right.$ cells per $\left.\mathrm{cm}^{2}\right)$ were seeded onto the scaffolds and cultured for $3 \mathrm{~d}$. Then the cell/scaffold constructs were transferred to a new tissue culture plates, washed with PBS and fixed using $2.5 \%$ glutaraldehyde for $24 \mathrm{~h}$. Then, the samples were washed with PBS again to remove glutaraldehyde and dehydrated with a series of gradient-increasing concentrations of ethanol (50\% for $60 \mathrm{~min}, 70 \%$ for $30 \mathrm{~min}, 80 \%$ for $20 \mathrm{~min}$, $90 \%$ for $10 \mathrm{~min}, 95 \%$ for $5 \mathrm{~min}$, and $100 \%$ for $5 \mathrm{~min}$ ). Finally, after dried in a natural air and coated with gold ( $90 \mathrm{~s}$ ), thus, the samples were observed by FESEM. $(n=3)$.

Laser scanning confocal microscopy (LSCM, Leica, Germany) was used to investigate cell spreading. Briefly, after cultured for $3 \mathrm{~d}$, the cell/scaffold constructs were washed by PBS and fixed using $4 \%$ neutral formaldehyde for $30 \mathrm{~min}$ at $37^{\circ} \mathrm{C}$. Thereafter, cells were washed by PBS again and immersed in $0.1 \%$ Triton X100 for 10 min to increase permeability. Then F-Actin Labeling Kit (AAT Bioquest, USA) and DAPI (Beyotime, China) were added and incubated for $60 \mathrm{~min}$ and $8 \mathrm{~min}$ at room temperature in a dark environment, respectively. Finally, after washed with PBS, the samples were observed by LSCM.

\section{Cell proliferation and differentiation}

Cell proliferation was evaluated by the Cell Counting Kit-8 assay (CCK-8, Dojindo, Japan) following the manufacturer's instructions. Specifically, the mBMSCs cells $\left(2 \times 10^{4}\right.$ cells per $\left.\mathrm{cm}^{2}\right)$ were seeded onto the scaffolds. At the pre-designed time points (1, 3, 5 days), the cell/scaffold constructs were transferred to a new plate. Then $350 \mathrm{~mL}$ of the CCK-8 working solution was added to each sample and incubated at $37{ }^{\circ} \mathrm{C}$ for $1 \mathrm{~h}$. Finally, $100 \mathrm{~mL}$ of the solution was pipetted to a 96-well plate and the absorbance at $450 \mathrm{~nm}$ was measured by using a microplate reader (Thermo Scientific 3001, USA). $(n=6)$.

The mBMSCs cells $\left(5 \times 10^{4}\right.$ cells per $\left.\mathrm{cm}^{2}\right)$ were seeded onto the $3 \mathrm{D}$ plotting scaffolds and cultured in osteogenic medium for 7 and $14 \mathrm{~d}$. Then the cell/scaffold constructs were transferred to a new culture plate for an alkaline phosphatase (ALP) staining assay and a real-time polymerase chain reaction (RT-PCR) test, respectively. The ALP staining assay was performed following the manufacturer's instructions using the BCIP/NBT phosphatase substrate (KPL, USA). $(n=3)$. The RT-PCR test was conducted to assay the gene expression. At the pre-designed time points $(7,14$ days), the cell/scaffold constructs were transferred to a new plate. Then they were washed by PBS and treated with trypsin $10 \mathrm{~min}$ to make cells suspended, thus, collecting the suspending cells for RT-PCR. Total RNA was extracted from six parallel samples using a Hi-Pure Total RNA Micro Kit (Megan Biological Technology, China). Total RNA concentrations were quantified using Nano Drop 2000 (Thermo Scientific, USA).
Then the RNA was reverse-transcribed into cDNA using a PrimeScript ${ }^{\mathrm{TM}}$ RT reagent kit (TaKaRa Biotechnology, Japan) according to the manufacturer's protocol. The RT-PCR was performed with the QuantStudio 6 Flex system (Life Technologies, USA) using the SYBR Green system (Genecopoeia, USA). Amplifications for cDNA samples were carried out at $95{ }^{\circ} \mathrm{C}$ for $10 \mathrm{~min}$, followed by 40 cycles at $95^{\circ} \mathrm{C}$ for $10 \mathrm{~s}, 60^{\circ} \mathrm{C}$ for $20 \mathrm{~s}$, and $72{ }^{\circ} \mathrm{C}$ for $15 \mathrm{~s}$. The following primer sequences were used: GAPDH gene: forward, 5'-TGGATGGCCCCTCCGGGAAA-3', reverse, 5'- AGTGGGGACACGGAAGGCCA-3'; RUNX2 gene: forward, 5'-CACTGGCGGTGCAACAAGA-3', reverse, 5'TTTCATAACAGCGGAGGCATTTC- ${ }^{\prime}$; ALP gene: forward, $5^{\prime}$ TGCCTACTTGTGTGGCGTGAA- ${ }^{\prime}$, reverse, $5^{\prime}$-TCACCCGAGTGGTAGTCACAATG-3' ${ }^{\prime}$; osteocalcin (OC) gene: forward, $5^{\prime}$ AGCAGCTTGGCCCAGACCTA- ${ }^{\prime}$, reverse, $5^{\prime}$-TAGCGCCGGAGTCTGTTCACTAC-3'; collagen I (Col I) gene: forward, $5^{\prime}$ TTCTGCTGCTAATGTTCTTGACC-3' ${ }^{\prime}$, reverse, $5^{\prime}$ - GGGATGAAGTATTGTGTCTTGGG-3'; osteopontin (OPN) gene: forward, $5^{\prime}$ TGCAAACACCGTTGTAACCAAAAGC- $3^{\prime}$, reverse, $5^{\prime}$ TCTTTACGTTTGCCGGTGACGT- $3^{\prime}$. The relative quantification of target genes was determined using glyceraldehyde 3-phosphate dehydrogenase (GAPDH) as a reference, and the $2^{-\Delta \Delta C_{t}}$ method was used to calculate the expression level of the target genes. Melting curve profiles were produced at the end of each PCR so as to confirm the specific transcriptions of amplification.

\section{Statistical analysis}

A one-way ANOVA followed by a Tukey test for means comparison was performed to assess the level of significance by employing the SPSS 19.0 statistics software. Results are expressed as the mean \pm standard error, and $p<0.05$ was designated as statistically significant.

\section{Results and discussion}

\section{Construction of 3D plotting scaffolds}

The Li-BCP and BCP scaffolds were successfully constructed by 3D plotting. It has been reported that BCP scaffolds can promote bone ingrowth and remodeling as well as vascularization. ${ }^{17}$ Specially, BCP was reported to induce bone formation in large, long bone defects in vivo when the weight ratio of $\mathrm{HA} / \beta$ TCP was $6: 4{ }^{31}$ Therefore, in this study, $6: 4$-weight-ratio HA/ $\beta$ TCP scaffolds were chosen as the control group. Besides, the pore size of scaffold was verified to be a key role in bone formation, however, there is no consensus about the optimum pore sizes that effectively facilitate bone ingrowth, some reports claimed that the ideal pore sizes for bone ingrowth were 100$200 \mu \mathrm{m}^{32,33}$ while others indicated that $250-500 \mu \mathrm{m}$ was better for proliferation and ECM production. ${ }^{34,35}$ In this work, we constructed the cylindrical Li-BCP and BCP scaffolds with a specification of $10 \mathrm{~mm} \times 10 \mathrm{~mm} \times 5 \mathrm{~mm}$ and a pore size of $200 \mu \mathrm{m}$ by $3 \mathrm{D}$ plotting technique.

\section{Characterization of scaffolds}

In this study, HA, $\beta$-TCP and Li- $\beta$-TCP as raw materials were used to construct 3D plotting scaffolds and their crystal phases 
were measured by XRD. As shown in Fig. 1(a) and (b), pure HA (JCPDS no. 72-1243), $\beta$-TCP and Li- $\beta$-TCP (JCPDS no. 09-0169) were successfully fabricated using wet chemical methods. The actual Li content in Li- $\beta$-TCP was tested by ICP and the results showed that $42.83 \%$ of theoretical Li content was incorporated into $\beta$-TCP successfully. (Theoretical concentration of $\mathrm{Li}$ was $5.708 \mathrm{mg} \mathrm{L}^{-1}$ while the actual concentration was $2.445 \mathrm{mg} \mathrm{L}^{-1}$ ). All these results demonstrated that raw materials used for 3D plotting scaffolds was pure and available. In addition, the consensus has been reached that there will be a phase transformation from $\beta$ to $\alpha$ when $\beta$-TCP is heated to some higher temperature. ${ }^{25}$ The crystal phases of BCP scaffolds under different sintering temperatures were also examined by XRD and the results were shown in Fig. 1(c). When the scaffolds were heated above $1150{ }^{\circ} \mathrm{C}$, for instance, at $1200{ }^{\circ} \mathrm{C}$ and $1250{ }^{\circ} \mathrm{C}$, the diffraction peaks of $\alpha$-TCP were detected. The crystal phases of BCP and Li-BCP scaffolds at $1150{ }^{\circ} \mathrm{C}$ can be seen in Fig. 1(d), there was no difference between the two scaffolds, which demonstrated that $1150{ }^{\circ} \mathrm{C}$ was the suitable sintering temperatures for both scaffolds.

The pore structure, morphology and microstructure of surface fiber were observed using SEM. As can be seen in Fig. 2(A), the fabricated scaffolds possessed of uniform geometric structure, and pore sizes were nearly $200 \mu \mathrm{m}$, which was consistent with the pre-designed pore size. Besides, the fiber arranged regularly with a crossing angel of $90^{\circ}$ and the pore structure slightly shrank because of a sintering process after construction of scaffolds. The cross-section images (Fig. 2(A)(c) and (f)) clearly showed a tight crossing between fibers, which may be contribute to enhance the mechanical strength of scaffolds. Overall, these results indicated that the constructed Li-BCP scaffold possessed of a well-proportioned pore structure and pore size.

The porosity and pore interconnectivity were also key factors in bone repair scaffolds and determined continuous ingrowth of bone tissue. ${ }^{3}$ In this study, micro-CT was conducted to identify the porosity and interconnectivity of scaffolds. As shown in Fig. 2(B), the results suggested that the Li-BCP and BCP scaffolds exhibited highly homogeneous pore structure. The pore interconnectivity and porosity of Li-BCP and BCP scaffolds were measured by mimics, and the results indicated that the addition of Li element into BCP scaffolds had negligible influence on the pore interconnectivity and the porosity. The pore interconnectivity of Li-BCP and BCP scaffolds were $99.98 \pm 0.002 \%$ and $99.96 \pm 0.013 \%$ respectively, while the porosity were $59.46 \pm$ $0.94 \%$ and $52.26 \pm 0.25 \%$, respectively. Previous studies had reported that incomplete pore interconnection would constrain the biological system and limited blood vessels invasion, ${ }^{36}$ vice
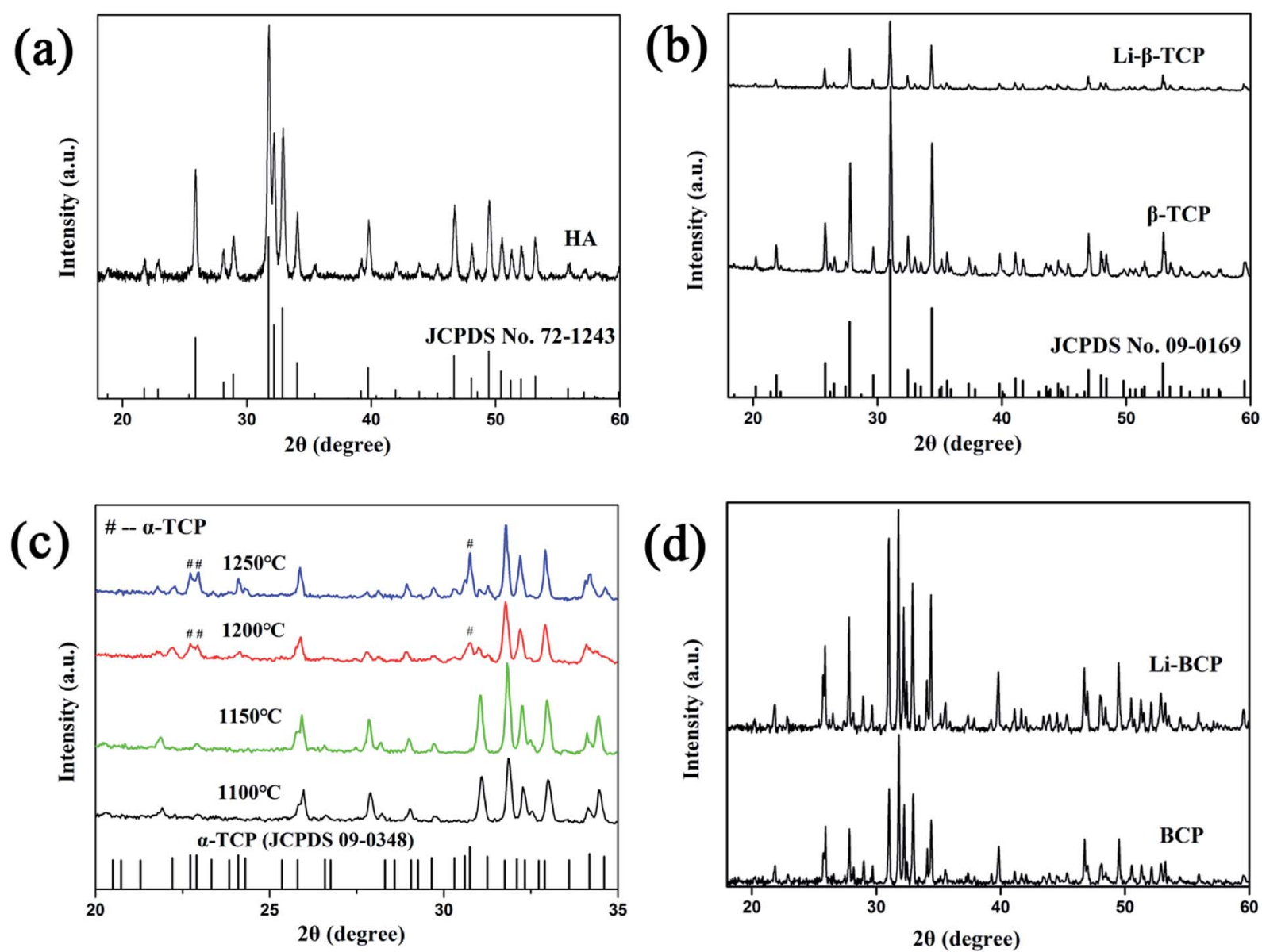

Fig. 1 XRD patterns of ( $a$ and b) HA, $\beta$-TCP, Li- $\beta$-TCP powder, (c) 3D plotting BCP scaffolds with different sintering temperatures and (d) 3D plotting BCP and Li-BCP scaffolds at $1150{ }^{\circ} \mathrm{C}$. 

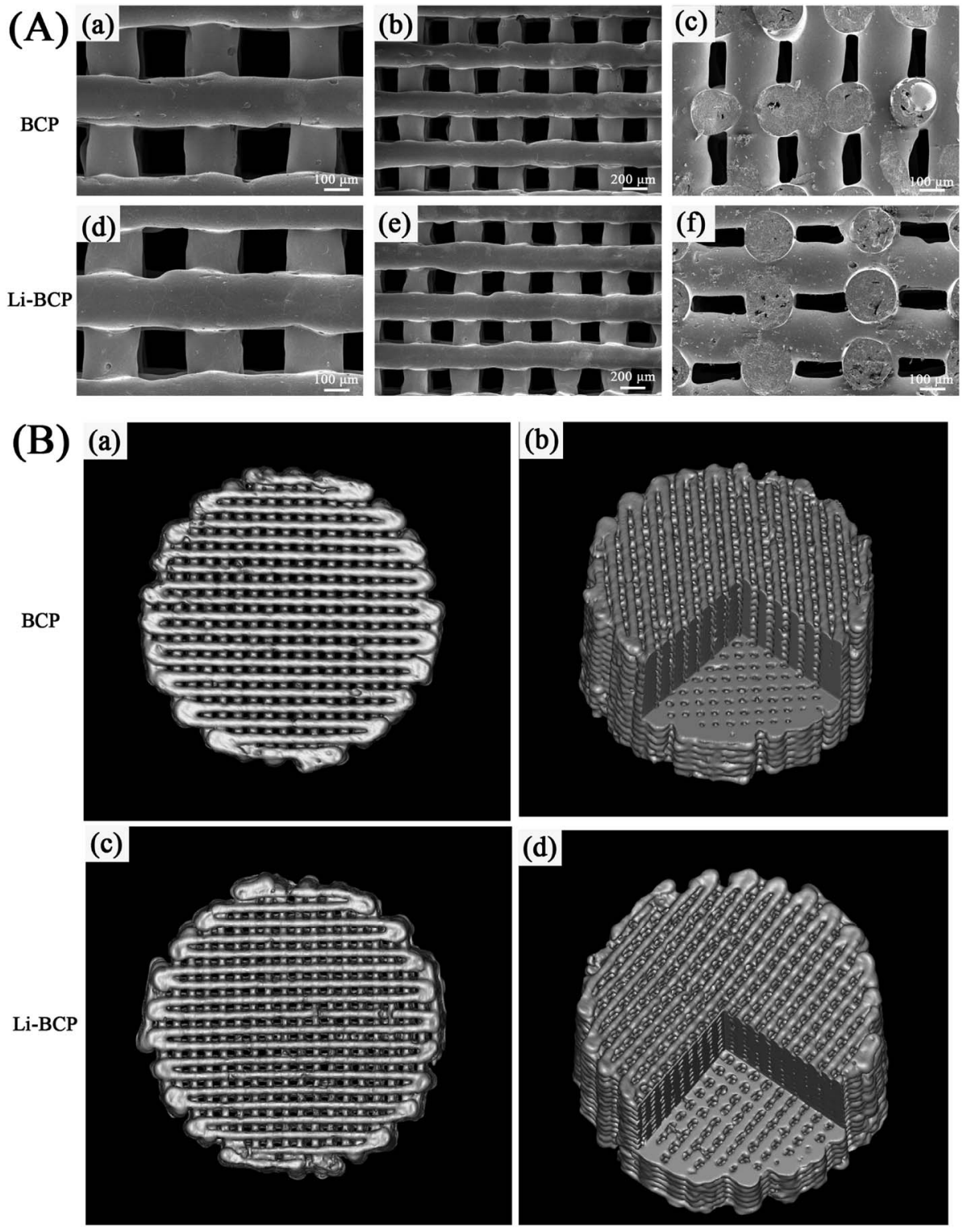

Fig. 2 (A) SEM images of 3D plotting BCP and Li-BCP scaffolds ((A): (a), (b), (d) and (e), top view images, (c) and (f), cross-section images) and (B) micro-CT images of 3D plotting BCP and Li-BCP scaffolds ((B): (a) and (c), top view images, (b) and (d), cross-section images).

versa, interconnections in porous ceramics would favor bone ingrowth inside the macropores. ${ }^{37}$ By the absence of reports, the titanium implants coating with $56-60 \%$ porosity by plasma spray could maximize bone ingrowth. ${ }^{38}$ All these above results demonstrated that the Li-containing BCP scaffolds possessed of an excellent pore interconnectivity and porosity, which contribute to the ingrowth of new bone tissue and vessels.

Mechanical strength is also an important parameter of scaffolds which defines a scaffold's performances and applications, especially, for bone repair. In our study, compressive strength and Young's modulus of the scaffolds were detected using universal material testing machine and the results were shown in Fig. 3. There were no distinct differences in mechanical strength between Li-BCP and BCP scaffolds, which demonstrated that the adulteration of $\mathrm{Li}$ would not affect the mechanical strength of scaffolds. The mean compressive strength of Li-BCP and BCP scaffolds were $27.09 \pm 3.33 \mathrm{MPa}$ and $29.21 \pm 3.63 \mathrm{MPa}$, respectively, and the mean Young's modulus were $724.88 \pm 23.99 \mathrm{MPa}$ and 803.75 \pm 65.55 $\mathrm{MPa}$, respectively. It had been reported that compressive modulus values of human trabecular bone range from 1 to $5000 \mathrm{MPa}$, with strength values ranging from 0.10 to $27.3 \mathrm{MPa} .{ }^{39}$ Therefore the Li-containing BCP scaffolds we designed could match the mechanical criterion of human trabecular bone and have great potential for bone repair.

\section{Cell adhesion and spreading}

Cell adhesion and spreading indicate the initial stages of cellscaffold interactions and subsequently influence cell proliferation and differentiation. ${ }^{40}$ In this study, we chose mBMSCs as model cells to assess the biological performance of the Li-BCP 

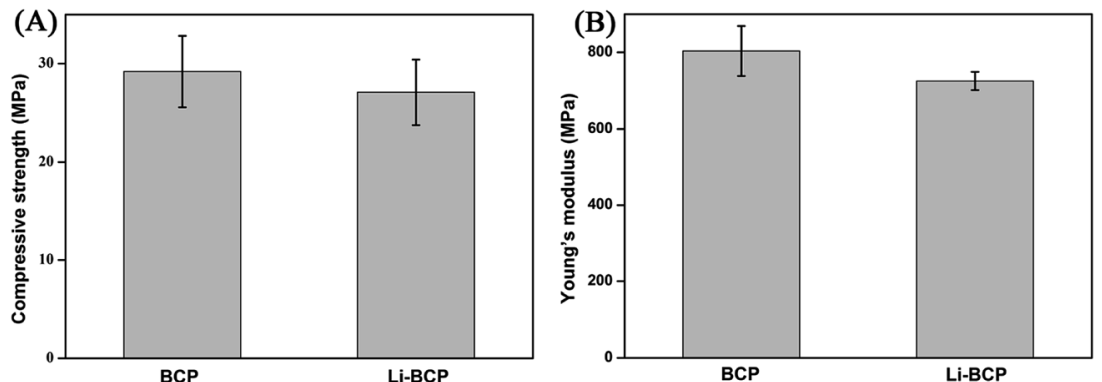

Fig. 3 Compressive strength and Young's modulus of 3D plotting BCP and Li-BCP scaffolds.

scaffolds. SEM was used to observe cell morphology and spread on $3 \mathrm{D}$ plotting scaffolds when mBMSCs were cultured for $3 \mathrm{~d}$. As can be seen in Fig. 4(A), mBMSCs spread well on both Li-BCP and BCP scaffolds, moreover, they presented stretched morphologies as well. Meanwhile, when mBMSCs were cultured on 3D plotting scaffolds for $5 \mathrm{~d}$, the cytoskeletons and cell nuclei were stained with F-actin (green) and DAPI (blue), respectively, then LSCM was used to investigate the morphology and adhesions of mBMSCs and the results were shown in Fig. 4(B), the mBMSCs cells were spread well on the Li-BCP scaffolds and the scaffolds were almost covered with cells, which demonstrated that 3D plotting Li-BCP scaffolds was excellent biocompatibility and favored of cell spreading.

\section{Cell proliferation and differentiation}

Cell proliferation was measured by CCK-8 assay after culturing mBMSCs on 3D plotting scaffolds for 1, 3 and $5 \mathrm{~d}$. As shown in Fig. 5, the results indicated that mBMSCs proliferated well on Li-BCP scaffolds and shared a similar growing trend with BCP
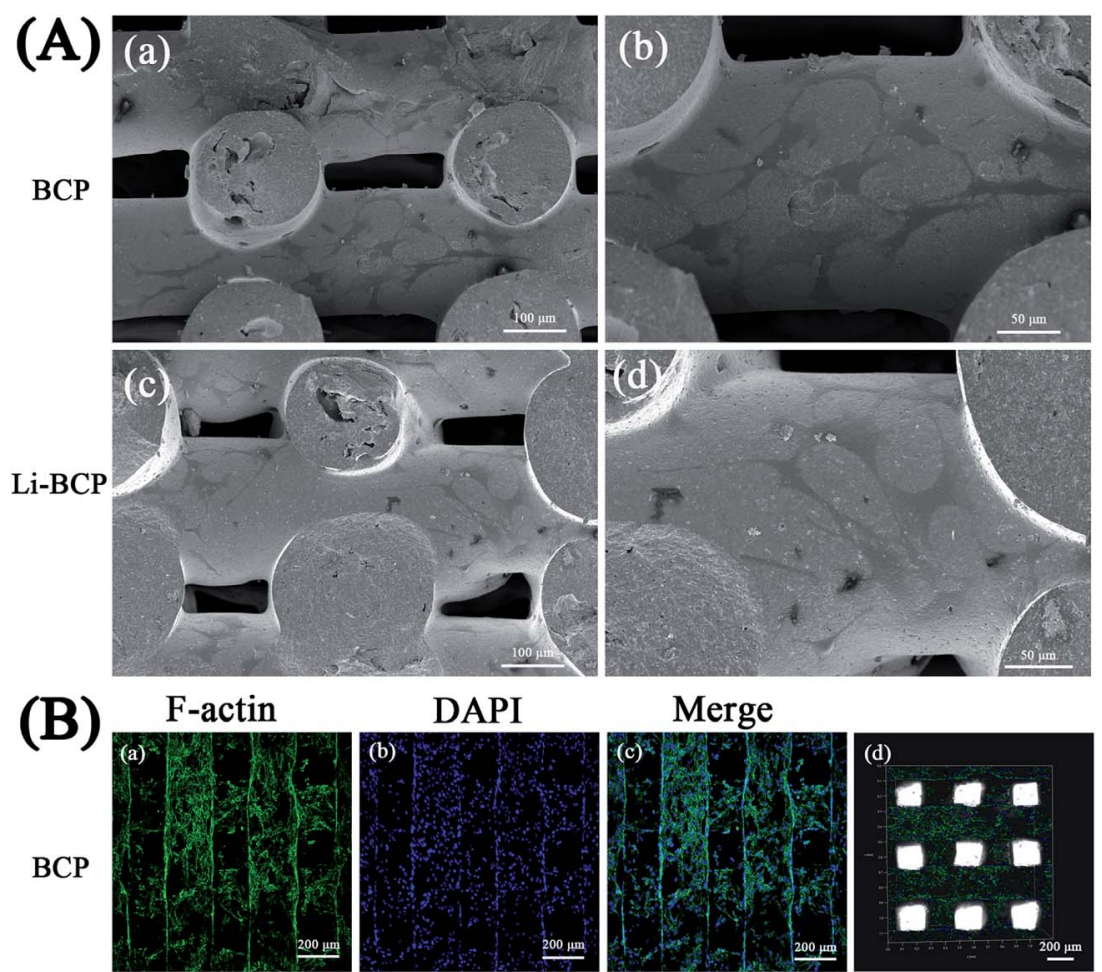

DAPI

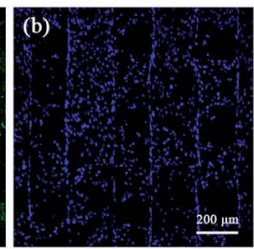

Merge
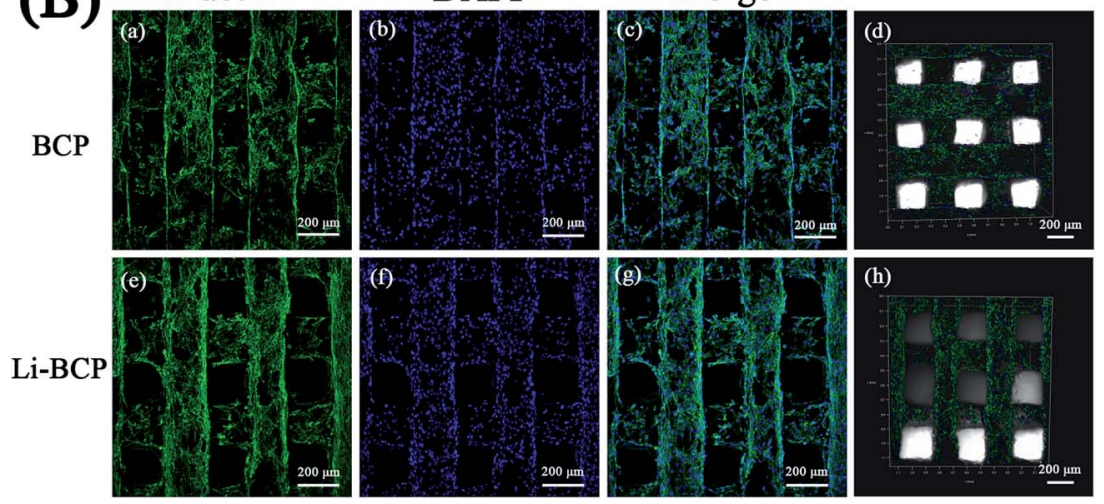

Fig. 4 (A) SEM images after culturing mBMSCs on 3D plotting BCP and Li-BCP scaffolds for $3 \mathrm{~d}((\mathrm{~A})$ : (b) and (d) were the enlarged views of (a) and (c)) and (B) LSCM images after culturing mBMSCs on 3D plotting BCP and Li-BCP scaffolds for $5 \mathrm{~d}((\mathrm{~B})$ : (d) and (h) were three-dimensional reconstruction images of $\mathrm{BCP}$ and $\mathrm{Li}-\mathrm{BCP}$ scaffolds). 


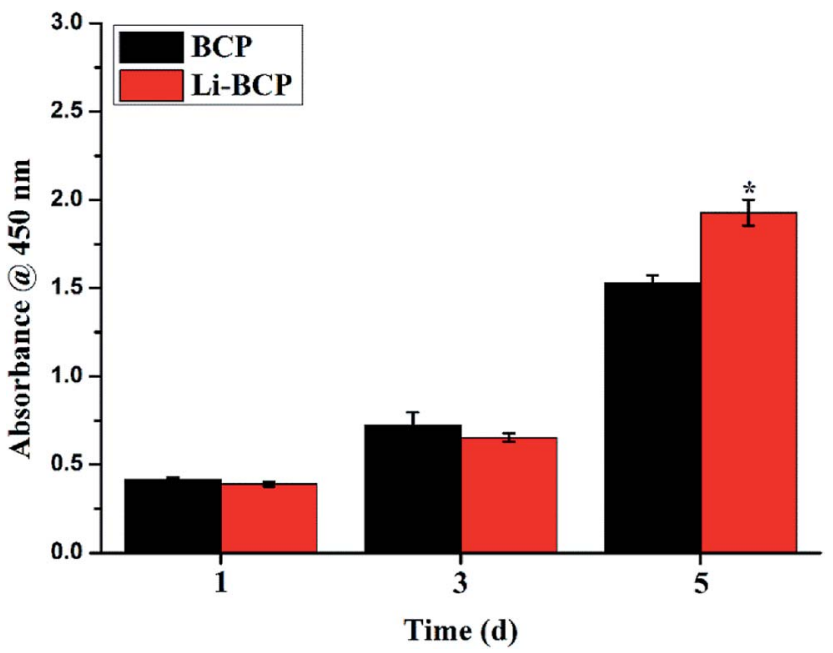

Fig. 5 Cell proliferation measured by CCK- 8 assay after culturing mBMSCs on 3D plotting Li-BCP and BCP scaffolds for 1,3 and $5 \mathrm{~d}$. The asterisk indicates that there was significant difference $(p<0.05)$.

scaffolds, which showed that Li-BCP scaffolds exhibited excellent bioactivity and biocompatibility. At 1 and $3 \mathrm{~d}$, there were no statistically significant differences between the Li-BCP and BCP scaffolds, which suggested that the growth rate of mBMSCs cells at initial stages kept synchronization. However, with the culture time prolonged to $5 \mathrm{~d}$, the growth rate of mBMSCs cells on LiBCP was higher than BCP scaffolds significantly, which demonstrated that Li-BCP scaffolds could enhance cell proliferation more effectively and possessed a higher cell growing rate than BCP scaffolds. The reason may be that the slow release of $\mathrm{Li}^{+}$inhibited glycogen synthase kinase, and then regulated the function of Cyclin D1 to affect the cell proliferation by activating the $\mathrm{Wnt} / \beta$-cetenin signaling pathway. ${ }^{41}$

The osteogenic differentiation of mBMSCs on 3D plotting scaffolds was also examined by detecting the protein and gene expression of alkaline phosphatase (ALP), which was known as an osteo-specific marker of bone metabolism, and the high expression of ALP implied the formation of active bone. ${ }^{42,43}$ ALP staining and RT-PCR were carried out to analyze the expression of ALP when mBMSCs were cultured for 7 and $14 \mathrm{~d}$ on the scaffolds. When the osteogenic differentiation of mBMSCs was seeded on the scaffolds, the scaffolds would be stained as bluepurple by BCIP/NBT assay, and the more blue-purple areas represented higher ALP activity and osteogenic differentiation ability. As can be seen in Fig. 6(A), when mBMSCs were cultured for 7 and $14 \mathrm{~d}$, there were a lot of blue-purple areas on the BCP and Li-BCP scaffolds. Besides, deeper blue-purple areas were found on Li-BCP scaffolds surfaces compared to those on BCP scaffolds, which demonstrated that Li-BCP scaffolds could promote the osteogenic differentiation of mBMSCs. With the culture time prolonging, more and deeper blue-purple areas were observed, which implied that the production of ALP increased gradually. In order to further testify the results of ALP staining, the quantitative expression of ALP gene was examined by RT-PCR. As shown in Fig. 6(B), the higher ALP expression level was found on Li-BCP compared to those of on BCP scaffolds on 7 and 14 day, which consistent with the results of ALP staining. Moreover, the expression of ALP gene increased with the culture time prolonging on both scaffolds. All these results demonstrated that Li-BCP scaffolds possessed a higher expression of ALP and osteogenic differentiation ability compared to BCP scaffolds.

Furthermore, another four key marker genes of osteogenic differentiation were also examined by RT-PCR, including RUNX2, Col I, OPN and OC. RUNX-2 is a multifunctional transcription factor that controls skeletal development and expresses at both early and late stage of osteogenic differentiation. ${ }^{\mathbf{4 4 , 4 5}} \mathrm{Col}$ I expresses at early stage of osteogenic differentiation. ${ }^{46}$ Both OPN and OC arise in the formation of mineralized bone-like nodules and expresses at the later period of bone differentiation. ${ }^{\mathbf{4 3}}$ These results of the relative expression of these genes when culturing mBMSCs on 3D plotting scaffolds for 7 and $14 \mathrm{~d}$ were analyzed by RT-PCR and the results were shown in Fig. 7. For RUNX-2, no statistically significant differences were reached at 7 days between the Li-BCP and BCP scaffolds. However, the expression on Li-BCP scaffolds was remarkably upregulated than that of BCP at 14 days. For Col I, a crosscurrent were observed at 7 and 14 days, highest expression level was presented for BCP scaffolds at 7 days while a contradictory trend at 14 day, which probably
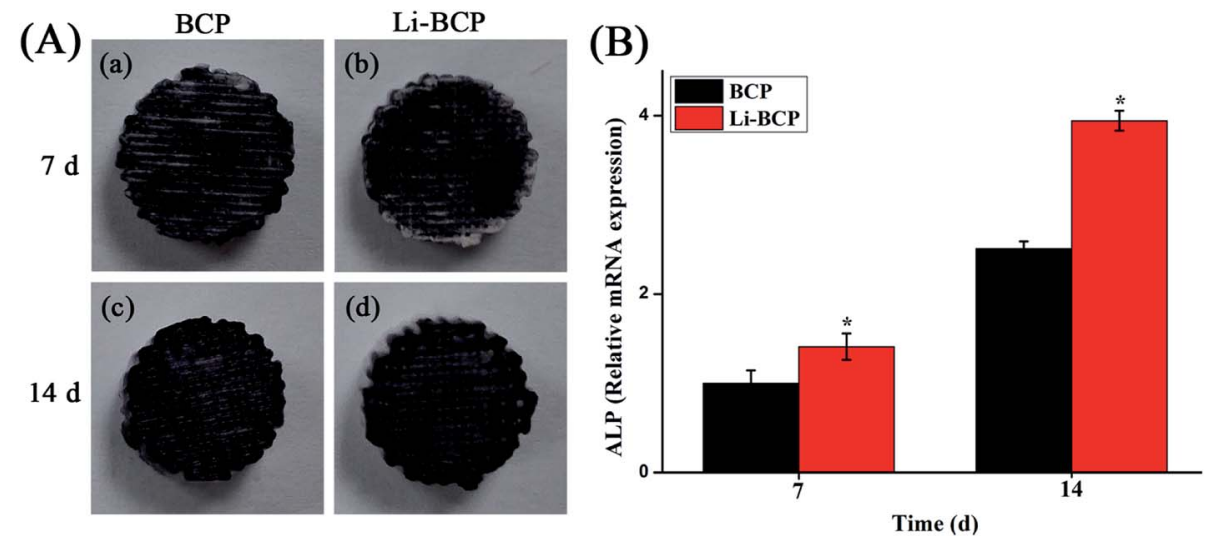

Fig. 6 (A) ALP staining and (B) ALP gene expression of mBMSCs on 3D plotting Li-BCP and BCP scaffolds after 7 and $14 \mathrm{~d}$ of culture. The asterisk indicates that there was significant difference $(p<0.05)$. 

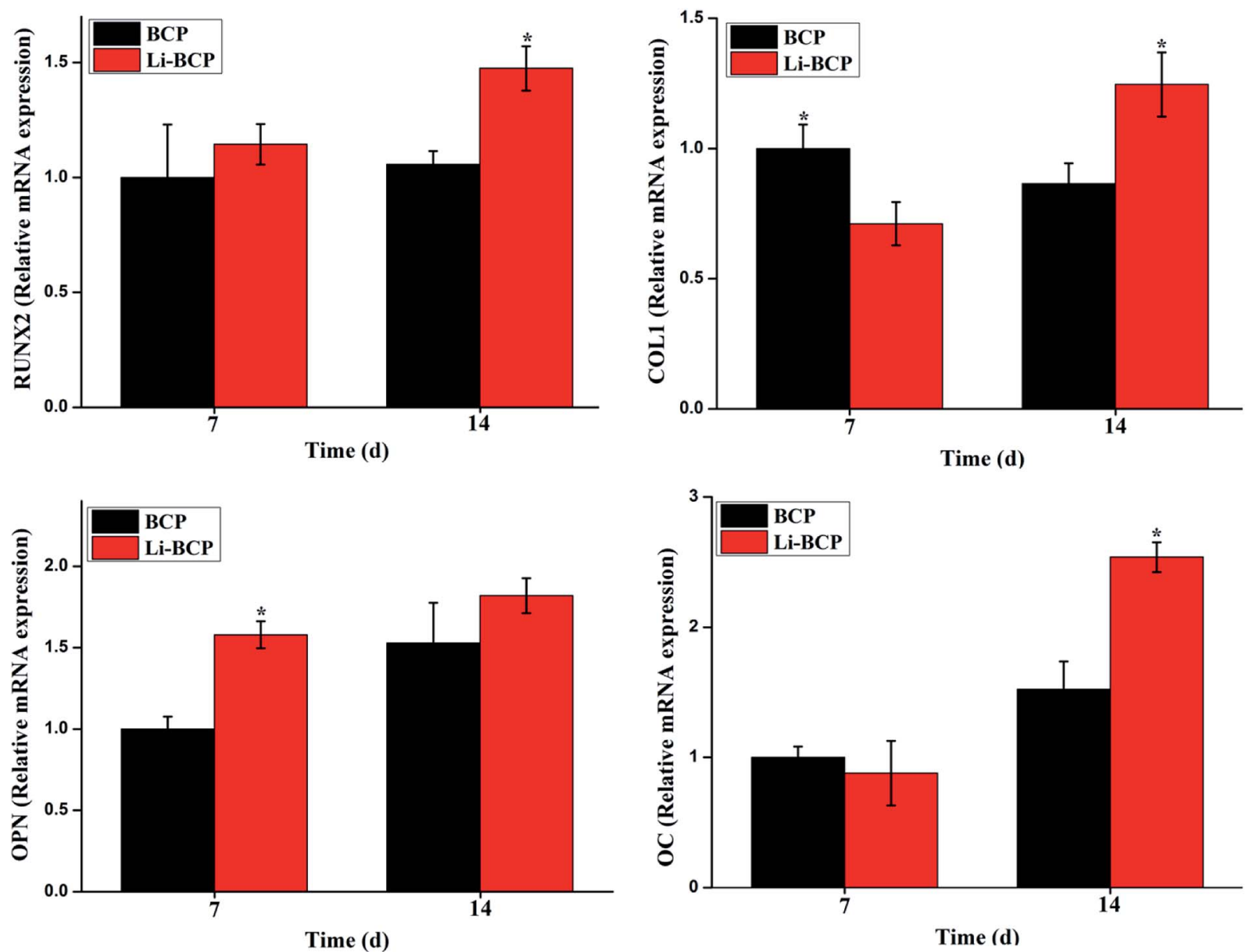

Fig. 7 Relative gene expression of runx-2, Col I, OPN and OC after cultured mBMSCs on 3D plotting Li-BCP and BCP scaffolds for 7 and 14 days. The asterisk indicates that there was significant difference $(p<0.05)$.

because BCP scaffolds could start osteogenic differentiation earlier, however, with the culture time extending, the expression of Col I on Li-BCP scaffolds exceeded the BCP scaffolds. For OPN, the expression was higher on Li-BCP scaffolds than that of on BCP at $7 \mathrm{~d}$, which demonstrated that Li-BCP scaffolds can enhance the osteogenic differentiation of mBMSCs at the early stage, while with the culture time prolong, there were no significant differences at $14 \mathrm{~d}$ between Li-BCP and BCP scaffolds. For OC, the expression was higher on Li-BCP than those of on $\mathrm{BCP}$ at $14 \mathrm{~d}$. These results demonstrated that Li-BCP scaffolds could enhance the mineralization at both early and late stage of osteogenic differentiation, and possessed more excellent osteogenic differentiation ability compared to BCP scaffolds. It is reasonable to speculate that $\mathrm{Li}$ ions released (see in the ESI, Table $1 \dagger$ ) from Li-BCP scaffolds can promote the osteogenic differentiation of mBMSCs. Previous reported that Li ions have a multidirectional effect and can induce the differentiation of different kinds of stem cells, such as BMSCs and PDLCs. ${ }^{23}$ Specially, Li ions can effectively induce osteogenic differentiation of stem cells by activating the Wnt signaling pathway. ${ }^{47}$ These results showed that the composition of $\mathrm{Li}^{+}$into BCP scaffolds can effectively enhance the activity of osteogenic differentiation compared to BCP scaffolds and had a great potential for bone repair scaffolds.

\section{Conclusions}

In summary, porous Li-BCP tissue engineering scaffolds were constructed successfully via the technique of 3D plotting. A series of characterizations was performed in detail, and the results indicated that the Li-BCP scaffolds constructed by 3D plotting technique possessed of welldesigned pore structure, pore sizes and excellent mechanical strength. More importantly, Li-BCP scaffolds could effectively enhance the proliferation and osteogenic differentiation of mBMSCs, which demonstrated that Li-BCP scaffolds could be seen as a potential candidate for bone repair applications.

\section{Acknowledgements}

This work was financially supported by the National Basic Research Program of China (Grant No. 2012CB619100), the National Natural Science Foundation of China (Grant No. 51232002, 51603074), the Science and Technology Program of Guangzhou city (Grant No. 201607010234), and Guangdong Natural Science Funds for Distinguished Young Scholar (Grant No. 2016A030306018). 


\section{Notes and references}

1 M. O. Wang, C. E. Vorwald, M. L. Dreher, E. J. Mott, M. H. Cheng, A. Cinar, H. Mehdizadeh, S. Somo, D. Dean, E. M. Brey and J. P. Fisher, Adv. Mater., 2015, 27, 138-144.

2 X. Xiao, W. Wang, D. Liu, H. Zhang, P. Gao, L. Geng, Y. Yuan, J. Lu and Z. Wang, Sci. Rep., 2015, 5, 9409.

3 S. Bose, S. Vahabzadeh and A. Bandyopadhyay, Mater. Today, 2013, 16, 496-504.

4 J.-H. Lee, S.-A. Park, K. Park, J.-H. Kim, K.-S. Kim, J. Lee and W. Kim, Chin. Sci. Bull., 2010, 55, 94-98.

5 H. N. Chia and B. M. Wu, J. Biol. Eng., 2015, 9, 4.

6 T. Pan, W. Song, X. Cao and Y. Wang, J. Mater. Sci. Technol., 2016, 32, 889-900.

7 P. Yilgor, R. A. Sousa, R. L. Reis, N. Hasirci and V. Hasirci, Macromol. Symp., 2008, 269, 92-99.

8 S. A. Park, S. H. Lee and W. D. Kim, Bioprocess Biosyst. Eng., 2011, 34, 505-513.

9 Y. Luo, C. Wu, A. Lode and M. Gelinsky, Biofabrication, 2013, 5, 015005.

10 G. Marchioli, L. van Gurp, P. P. van Krieken, D. Stamatialis, M. Engelse, C. A. van Blitterswijk, M. B. Karperien, E. de Koning, J. Alblas, L. Moroni and A. A. van Apeldoorn, Biofabrication, 2015, 7, 025009.

11 A. R. Akkineni, Y. Luo, M. Schumacher, B. Nies, A. Lode and M. Gelinsky, Acta Biomater., 2015, 27, 264-274.

12 K. Haberstroh, K. Ritter, J. Kuschnierz, K. H. Bormann, C. Kaps, C. Carvalho, R. Mulhaupt, M. Sittinger and N. C. Gellrich, J. Biomed. Mater. Res., Part B, 2010, 93, 520530.

13 Y. Luo, A. Lode, A. R. Akkineni and M. Gelinsky, RSC Adv., 2015, 5, 43480-43488.

14 Y. Luo, A. Lode, C. Wu, J. Chang and M. Gelinsky, ACS Appl. Mater. Interfaces, 2015, 7, 6541-6549.

15 Y. Luo, A. Lode, F. Sonntag, B. Nies and M. Gelinsky, J. Mater. Chem. B, 2013, 1, 4088-4098.

16 I. H. Liu, S. H. Chang and H. Y. Lin, Biomed. Mater., 2015, 10, 035004.

17 M. Castilho, C. Moseke, A. Ewald, U. Gbureck, J. Groll, I. Pires, J. Teßmar and E. Vorndran, Biofabrication, 2014, 6, 015006.

18 D. Tadic and M. Epple, Biomaterials, 2004, 25, 987-994.

19 R. Detsch, S. Schaefer, U. Deisinger, G. Ziegler, H. Seitz and B. Leukers, J. Biomater. Appl., 2011, 26, 359-380.

20 S. V. Dorozhkin, Acta Biomater., 2012, 8, 963-977.

21 O. Malard, J. Guicheux, J.-M. Bouler, O. Gauthier, C. Beauvillain de Montreuil, E. Aguado, P. Pilet, R. LeGeros and G. Daculsi, Bone, 2005, 36, 323-330.

22 O. Gauthier, J. M. Bouler, E. Aguado, R. Z. Legeros, P. Pilet and G. Daculsi, J. Mater. Sci.: Mater. Med., 1999, 10, 199-204.

23 P. Han, M. Xu, J. Chang, N. Chakravorty, C. Wu and Y. Xiao, Biomater. Sci., 2014, 2, 1230.
24 N. Matsumoto, K. Yoshida, K. Hashimoto and Y. Toda, Mater. Res. Bull., 2009, 44, 1889-1894.

25 S. Tarafder, N. M. Davies, A. Bandyopadhyay and S. Bose, Biomater. Sci., 2013, 1, 1250-1259.

26 W. Xue, K. Dahlquist, A. Banerjee, A. Bandyopadhyay and S. Bose, J. Mater. Sci.: Mater. Med., 2008, 19, 2669-2677.

27 G. Fielding and S. Bose, Acta Biomater., 2013, 9, 9137-9148.

28 M. Tian, F. Chen, W. Song, Y. Song, Y. Chen, C. Wan, X. Yu and X. Zhang, J. Mater. Sci.: Mater. Med., 2009, 20, 15051512.

29 S. Bose, G. Fielding, S. Tarafder and A. Bandyopadhyay, Trends Biotechnol., 2013, 31, 594-605.

30 A. Zamani, G. R. Omrani and M. M. Nasab, Bone, 2009, 44, 331-334.

31 T. L. Arinzeh, T. Tran, J. McAlary and G. Daculsi, Biomaterials, 2005, 26, 3631-3638.

32 V. Karageorgiou and D. Kaplan, Biomaterials, 2005, 26, 54745491.

33 A. C. Jones, C. H. Arns, A. P. Sheppard, D. W. Hutmacher, B. K. Milthorpe and M. A. Knackstedt, Biomaterials, 2007, 28, 2491-2504.

34 L. Yang, K. Tanabe, T. Miura, M. Yoshinari, S. Takemoto, S. Shintani and M. Kasahara, Dent. Mater. J., 2017, DOI: 10.4012/dmj.2016-242.

35 L. Polo-Corrales, M. Latorre-Esteves and J. E. Ramirez-Vick, J. Nanosci. Nanotechnol., 2014, 14, 15-56.

36 M. Mastrogiacomo, S. Scaglione, R. Martinetti, L. Dolcini, F. Beltrame, R. Cancedda and R. Quarto, Biomaterials, 2006, 27, 3230-3237.

37 J. X. Lu, B. Flautre, K. Anselime and P. Hardouin, J. Mater. Sci.: Mater. Med., 1999, 10, 111-120.

38 V. Karageorgiou and D. Kaplan, Biomaterials, 2005, 26, 54745491.

39 J. M. Williams, A. Adewunmi, R. M. Schek, C. L. Flanagan, P. H. Krebsbach, S. E. Feinberg, S. J. Hollister and S. Das, Biomaterials, 2005, 26, 4817-4827.

40 J. Song, H. Gao, G. Zhu, X. Cao, X. Shi and Y. Wang, Biofabrication, 2016, 8, 035009.

41 E. M. Wexler, D. H. Geschwind and T. D. Palmer, Mol. Psychiatry, 2008, 13, 285-292.

42 J. Song, H. Gao, G. Zhu, X. Cao, X. Shi and Y. Wang, Carbon, 2015, 95, 1039-1050.

43 L. Hao, H. Yang, C. Du, X. Fu, N. Zhao, S. Xu, F. Cui, C. Mao and Y. Wang, J. Mater. Chem. B, 2014, 2, 4794-4801.

44 M. H. Lee, Y. J. Kim, H. J. Kim, H. D. Park, A. R. Kang, H. M. Kyung, J. H. Sung, J. M. Wozney, H. J. Kim and H. M. Ryoo, J. Biol. Chem., 2003, 278, 34387-34394.

45 T. Komori, Cell Tissue Res., 2009, 339, 189-195.

46 F. Zou, N. Zhao, X. Fu, J. Diao, Y. Ma, X. Cao, S. Wan, S. Zhong and Y. Wang, RSC Adv., 2016, 6, 23588-23598.

47 C. Y. Logan and R. Nusse, Annu. Rev. Cell Dev. Biol., 2004, 20, 781-810. 